\title{
MATRICES OF ZEROS AND ONES
}

\author{
H. J. RYSER ${ }^{1}$
}

Let $A$ be a matrix of $m$ rows and $n$ columns and let the entries of $A$ be the integers 0 and 1 . We call such a matrix a $(0,1)$-matrix of size $m$ by $n$. The $2^{m n}(0,1)$-matrices of size $m$ by $n$ play a fundamental role in a wide variety of combinatorial investigations. One of the chief reasons for this is the following. Let $X$ be a set of $n$ elements $x_{1}, x_{2}, \cdots, x_{n}$ and let $X_{1}, X_{2}, \cdots, X_{m}$ be $m$ subsets of $X$. Let $a_{i j}=1$ if $x_{j}$ is a member of $X_{i}$ and let $a_{i j}=0$ if $x_{j}$ is not a member of $X_{i}$. The $a_{i j}$ 's yield a $(0,1)$-matrix $A=\left[a_{i j}\right]$ of size $m$ by $n$ called the incidence matrix for the subsets $X_{1}, X_{2}, \cdots, X_{m}$ of $X$. The 1 's in row $i$ of $A$ specify the elements that belong to set $X_{i}$ and the 1's in column $j$ of $A$ specify the sets that contain element $x_{j}$. The matrix $A$ characterizes the $m$ subsets $X_{1}, X_{2}, \cdots, X_{m}$ of the set $X$.

Let $A$ be a $(0,1)$-matrix of size $m$ by $n$. Let the sum of row $i$ of $A$ be denoted by $r_{i}$ and let the sum of column $j$ of $A$ be denoted by $s_{j}$. We call

$$
R=\left(r_{1}, r_{2}, \cdots, r_{m}\right)
$$

the row sum vector and

$$
S=\left(s_{1}, s_{2}, \cdots, s_{n}\right)
$$

the column sum vector of $A$. If $\tau$ denotes the total number of 1 's in $A$, then it is clear that

$$
\tau=\sum_{i=1}^{m} r_{i}=\sum_{j=1}^{n} s_{j}
$$

The vectors $R$ and $S$ determine a class

$$
\mathfrak{A}=\mathfrak{A}(R, S),
$$

consisting of all $(0,1)$-matrices of size $m$ by $n$ with row sum vector $R$ and column sum vector $S$. In this paper we summarize portions of the extensive literature on $(0,1)$-matrices and give special emphasis to problems dealing with the class $\mathfrak{A}(R, S)$. We discuss diversified topics including traces, term ranks, widths, heights, and combinatorial designs. A good deal of the subject matter is still in its infancy

An address delivered before the Detroit meeting of the Society on November 27, 1959 , by invitation of the Committee to Select Hour Speakers for Western Sectional Meetings; received by the editors June 22, 1960.

1 The present research was supported in part by the Office of Ordnance Research. 
and throughout the paper we mention a number of unsettled questions. A rather lengthy but by no means complete bibliography appears at the end of the paper.

1. The class $\mathfrak{A}(R, S)$. Let $R=\left(r_{1}, r_{2}, \cdots, r_{m}\right)$ and $S=\left(s_{1}, s_{2}, \cdots, s_{n}\right)$ be two vectors whose components are nonnegative integers. Let $\mathfrak{A}(R, S)$ denote the class of all $(0,1)$-matrices of size $m$ by $n$ with row sum vector $R$ and column sum vector $S$. We describe a simple necessary and sufficient condition on the vectors $R$ and $S$ in order that the class $\mathfrak{A}(R, S)$ be nonempty. Let

$$
\delta_{i}=(1,1, \cdots, 1,0,0, \cdots, 0) \quad(i=1,2, \cdots, m)
$$

be a vector of $n$ components with 1 's in the first $r_{i}$ positions and 0 's elsewhere. A matrix of the form

$$
\bar{A}=\left[\begin{array}{c}
\delta_{1} \\
\delta_{2} \\
\vdots \\
\delta_{m}
\end{array}\right]
$$

is called maximal and we refer to $\bar{A}$ as the maximal matrix with row sum vector $R$. Let $\bar{S}=\left(\bar{s}_{1}, \bar{s}_{2}, \cdots, \bar{s}_{n}\right)$ be the column sum vector of $\bar{A}$. Then

$$
\sum_{i=1}^{m} r_{i}=\sum_{j=1}^{n} \bar{s}_{j}
$$

are conjugate partitions and the class $\mathfrak{A}(R, \bar{S})$ consists of the single matrix $\bar{A}$. Let $S=\left(s_{1}, s_{2}, \cdots, s_{n}\right)$ and $S^{*}=\left(s_{1}^{*}, s_{2}^{*}, \cdots, s_{n}^{*}\right)$ be two vectors whose components are nonnegative integers. The vector $S$ is majorized by $S^{*}[32 ; 46]$

$$
S<S^{*}
$$

provided that with subscripts renumbered

(1.5) $s_{1} \geqq s_{2} \geqq \cdots \geqq s_{n}$

$$
s_{1}^{*} \geqq s_{2}^{*} \geqq \cdots \geqq s_{n}^{*},
$$

(1.6) $s_{1}+s_{2}+\cdots+s_{i} \leqq s_{1}^{*}+s_{2}^{*}+\cdots+s_{i}^{*}$

$$
(i=1,2, \cdots, n-1) \text {, }
$$

(1.7) $s_{1}+s_{2}+\cdots+s_{n}=s_{1}^{*}+s_{2}^{*}+\cdots+s_{n}^{*}$.

We are now in a position to state the conditions under which the class $\mathfrak{A}(R, S)$ is nonempty. 
TheOREM 1.1. Let $R=\left(r_{1}, r_{2}, \cdots, r_{m}\right)$ and $S=\left(s_{1}, s_{2}, \cdots, s_{n}\right)$ be two vectors whose components are nonnegative integers. Let $\bar{A}$ be the maximal matrix of size $m$ by $n$ with row sum vector $R$ and column sum vector $\bar{S}$. Then a necessary and sufficient condition in order that the class $\mathfrak{A}(R, S)$ be nonempty is that

$$
S<\bar{S} \text {. }
$$

The necessity of Theorem 1.1 is obvious. Proofs of the sufficiency appear in [17] and [58]. An application of Theorem 1.1 involving the completion of $r$ by $s$ latin rectangles to $n$ by $n$ latin squares is investigated in [58]. Generalizations and a critical survey of a wide range of related topics are discussed in [34]. A difficult problem requires the determination of the precise number of matrices in $\mathfrak{A}(R, S)$. This has been studied but suffice it to say the number of matrices in $\mathfrak{U}(R, S)$ is an exceedingly intricate function of $R$ and $S$ [67]. Further insight here would be of considerable interest. In what follows we always assume that the class $\mathfrak{A}(R, S)$ is nonempty.

Let $A$ be a matrix in $\mathfrak{A}(R, S)$. Consider the 2 by 2 submatrices of $A$ of the types

$$
A_{1}=\left[\begin{array}{ll}
1 & 0 \\
0 & 1
\end{array}\right] \text { and } A_{2}=\left[\begin{array}{ll}
0 & 1 \\
1 & 0
\end{array}\right]
$$

An interchange is a transformation of the elements of $A$ that changes a minor of type $A_{1}$ into type $A_{2}$ or vice versa and leaves all other elements of $A$ unaltered [58]. This is, in a sense, the most elementary operation that may be applied to $A$ to yield a new matrix in the class $\mathfrak{U}(R, S)$. The interchange concept gives us a simple procedure for the construction of a matrix in $\mathfrak{U}(R, S)[17 ; 19]$. Let $A$ be a matrix in $\mathfrak{U}(R, S)$. Let $R_{1}$ be a row vector of $r_{1} 1$ 's and $n-r_{1} 0$ 's. Let the 1 's be inserted in the positions in which $S$ has its $r_{1}$ largest components. Let $R_{2}$ be a row vector of $r_{2} 1$ 's and $n-r_{2} 0$ 's. Let the 1 's be inserted in the positions in which $S-R_{1}$ has its $r_{2}$ largest components. $R_{3}$ is a row vector whose 1's are in the positions in which $S-R_{1}-R_{2}$ has its $r_{3}$ largest components, and so on. Let

$$
A_{R}=\left[\begin{array}{c}
R_{1} \\
R_{2} \\
\vdots \\
\dot{R}_{m}
\end{array}\right] .
$$

It is clear that $A_{R}$ may be constructed directly from the vectors $R$ and $S$. We prove that $A_{R}$ belongs to $\mathfrak{A}(R, S)$. We may apply inter- 
changes to $A$ and replace row 1 of $A$ by $R_{1}$. Then we may apply interchanges to the transformed matrix and replace row 2 by $R_{2}$. These interchanges do not involve $R_{1}$. In this way we transform $A$ into $A_{R}$ by interchanges. But then $A_{R}$ has row sum vector $R$ and column sum vector $S$ and hence $A_{R}$ belongs to $\mathfrak{A}(R, S)$. An analogous construction by columns of a matrix $A_{S}$ may be carried out by interchanging the roles of $R$ and $S$ in the preceding discussion.

Theorem 1.2. Let $A$ and $A^{\prime}$ belong to $\mathfrak{X}(R, S)$. Then $A$ is transformable into $A^{\prime}$ by a finite sequence of interchanges.

This theorem is the interchange theorem [58]. It is helpful in dealing with many problems involving the class $\mathfrak{A}(R, S)$. We give a simple proof based on the matrix $A_{R}$ of (1.8) [19]. We may transform $A$ into $A_{R}$ and $A^{\prime}$ into $A_{R}$ by finite sequences of interchanges. Let the intermediate matrices taking $A^{\prime}$ into $A_{R}$ be $A_{1}, A_{2}, \cdots, A_{q}$. But then there exists an interchange taking $A_{R}$ into $A_{q}$. Also there exists an interchange taking $A_{q}$ into $A_{q-1}$ and so on. Thus $A_{R}$ is transformable into $A^{\prime}$ by interchanges and hence $A$ is transformable into $A^{\prime}$ by interchanges.

2. The structure matrix. Let $A$ be a matrix in the class $\mathfrak{A}(R, S)$. In many investigations one assumes without loss of generality that the row sum vector $R$ and the column sum vector $S$ of $A$ satisfy

$$
\begin{aligned}
& r_{1} \geqq r_{2} \geqq \cdots \geqq r_{m}>0, \\
& s_{1} \geqq s_{2} \geqq \cdots \geqq s_{n}>0 .
\end{aligned}
$$

This means that we have excluded zero rows and zero columns and permuted rows and columns so that they are nonincreasing. A nonempty class $\mathfrak{A}(R, S)$ with $R$ and $S$ satisfying (2.1) and (2.2) is called normalized. Henceforth throughout our discussion we take $\mathfrak{H}(R, S)$ normalized.

Let $A$ belong to the normalized class $\mathfrak{A}(R, S)$ and write

$$
A=\left[\begin{array}{cc}
W & X \\
Y & Z
\end{array}\right]
$$

where $W$ is of size $e$ by $f(0 \leqq e \leqq m ; 0 \leqq f \leqq n)$. Let $Q$ be a $(0,1)$-matrix and let $N_{0}(Q)$ denote the number of 0 's in $Q$ and let $N_{1}(Q)$ denote the number of 1 's in $Q$. Now let

$$
t_{e f}=N_{0}(W)+N_{1}(Z) \quad(e=0,1, \cdots, m ; f=0,1, \cdots, n) .
$$

We call the $m+1$ by $n+1$ matrix

$$
T=\left[t_{e f}\right]
$$


the structure matrix of the normalized class $\mathfrak{A}(R, S)$ [62]. An easy calculation shows that

$$
t_{e f}=e f+\left(r_{e+1}+r_{e+2}+\cdots+r_{m}\right)-\left(s_{1}+s_{2}+\cdots+s_{f}\right) .
$$

Thus the structure matrix is independent of the particular choice of $A$ in $\mathfrak{A}(R, S)$. From (2.6) it follows without difficulty that

$$
\begin{aligned}
t_{e, f+1} & =t_{e f}+e-s_{f+1} \\
& (e=0,1, \cdots, m ; f=0,1, \cdots, n-1)
\end{aligned}
$$

and

$$
\begin{aligned}
& t_{e+1, f}=t_{e f}+f-r_{e+1} \\
& \quad(e=0,1, \cdots, m-1 ; f=0,1, \cdots, n) .
\end{aligned}
$$

The recursions (2.7) and (2.8) are useful in constructing $T$ from a given $R$ and $S$.

The structure matrix $T$ contains a wealth of information concerning the class $\mathfrak{A}(R, S)$. This will be apparent in succeeding sections. The entries of $T$ are, of course, nonnegative integers and its size is $m+1$ by $n+1$. For notational convenience we number the rows of $T$ from 0 through $m$ and its columns from 0 through $n$. Let $E_{k}$ be the triangular matrix of order $k+1$ with 1 's on and below the main diagonal and 0's elsewhere and let $E_{k}^{T}$ denote the transpose of $E_{k}$. Let $\tau$ denote the total number of 1 's in a matrix $A$ of $\mathfrak{A}(R, S)$ and let $J$ be the $m$ by $n$ matrix with all entries equal to 1 . Then by direct calculation it may be verified that

$$
E_{m}\left[\begin{array}{ccc}
\tau & -s_{1} & -s_{2} \cdots-s_{n} \\
-r_{1} & \\
-r_{2} & J \\
\vdots &
\end{array}\right] E_{n}^{T}=T .
$$

One may also deduce that if $e \leqq s_{n}$, then

$$
t_{e 0} \geqq t_{e 1} \geqq \cdots \geqq t_{e n},
$$

and if $e \geqq s_{1}$, then

$$
t_{e 0} \leqq t_{e 1} \leqq \cdots \leqq t_{e n} .
$$

However, if $s_{n}<e<s_{1}$, then there exists an integer $f(0<f<n)$ such that

$$
t_{e 0} \geqq t_{e 1} \geqq \cdots \geqq t_{e f} \leqq t_{e, f+1} \leqq \cdots \leqq t_{e n} .
$$


The columns of $T$ have the analogous monotonic behavior. The following example illustrates the preceding remarks:

$$
\begin{aligned}
R & =S=(3,3,1,1) \\
T & =\left[\begin{array}{llllll}
8 & 5 & 2 & 1 & 0 \\
5 & 3 & 1 & 1 & 1 \\
2 & 1 & 0 & 1 & 2 \\
1 & 1 & 1 & 3 & 5 \\
0 & 1 & 2 & 5 & 8
\end{array}\right] .
\end{aligned}
$$

We now give an example of how the structure matrix $T$ reveals properties of the class $\mathfrak{A}(R, S)$. Let $A$ belong to the normalized class $\mathfrak{U}(R, S)$. An element $a_{e f}=1$ of $A$ is an invariant 1 provided that no sequence of interchanges applied to $A$ replaces $a_{e f}=1$ by 0 . If $a_{e f}=1$ is an invariant 1 of $A$, then by the interchange theorem the entries in the $(e, f)$ position of all of the matrices in $\mathfrak{A}(R, S)$ must be invariant 1 's. Thus all or none of the matrices in $\mathfrak{A}(R, S)$ contain invariant 1 's and we say $\mathfrak{A}(R, S)$ is with or without invariant 1's.

One may deduce the following [59].

THEOREM 2.1. The normalized class $\mathfrak{Y}(R, S)$ is with invariant 1's if and only if the matrices in $\mathfrak{Y}(R, S)$ are of the form

$$
A=\left[\begin{array}{ll}
J & * \\
* & 0
\end{array}\right] .
$$

Here $J$ is a matrix of 1 's of specified size e by $f(0<e \leqq m ; 0<f \leqq n)$ and 0 is a zero matrix.

Consider now the structure matrix $T$. We have called the first row and column of $T$ the 0 th row and column of $T$. Then by Theorem $2.1 \mathfrak{H}(R, S)$ is with invariant 1 's if and only if a 0 entry appears among rows $1,2, \cdots, m$ and columns $1,2, \cdots, n$ of $T$. In fact the position of each such 0 in $T$ gives appropriate integers $e$ and $f$ for the decomposition (2.10). In our example with $R=S=(3,3,1,1)$, the class $\mathfrak{Y}(R, S)$ is with invariant 1's and $e=f=2$.

3. Traces. In this and in the following two sections we associate combinatorically significant integers with each of the matrices in the normalized class $\mathfrak{U}(R, S)$ and study the maximum and minimum of these integers over the matrices of the class. We begin with the trace. For a $(0,1)$-matrix $A$ of size $m$ by $n$ the trace of $A$ is defined by

$$
\sigma=\sum_{i=1}^{t} a_{i i}, \quad t=\min (m, n) .
$$


If $A$ is the incidence matrix for the subsets $X_{1}, X_{2}, \cdots, X_{m}$ of $X$, then the trace counts the number of times that $x_{i}$ is a member of $X_{i}$ for $i=1,2, \cdots, t$. Now let $A$ be a matrix of the normalized class $\mathfrak{A}(R, S)$ and let $\bar{\sigma}$ denote the maximal and $\tilde{\sigma}$ the minimal trace of the matrices in $\mathfrak{A}(R, S)$. One may deduce the following [62].

Theorem 3.1. Let $\bar{\sigma} \neq \min (m, n)$. Then there exists a matrix $A_{\bar{\sigma}}$ of trace $\bar{\sigma}$ in the normalized class $\mathfrak{A}(R, S)$ of the form

$$
A_{\bar{\sigma}}=\left[\begin{array}{ccc}
J & * & * \\
* & \tilde{0} & 0 \\
* & 0 & 0
\end{array}\right] .
$$

Here $J$ is a matrix of 1 's of specified size e by $f(0<e \leqq \bar{\sigma} ; 0<f \leqq \bar{\sigma})$. The matrix 0 is of size $g$ by $h$ and has 1's in the main diagonal positions of $A_{\bar{\sigma}}$ and 0 's in all other positions. Moreover,

$$
e+g=f+h=\bar{\sigma} .
$$

The 0's denote zero matrices.

Theorem 3.1 is derived by interchange manipulations that exploit the maximal property of $\bar{\sigma}$. If in a $(0,1)$-matrix the 0 's are replaced by 1 's and the 1 's by 0 's, then the resulting matrix is called the complement of the original matrix. The analogue of Theorem 3.1 for the minimal trace $\tilde{\sigma}$ may be derived from Theorem 3.1 by an investigation of complements [62].

Theorem 3.2. Let the matrices in the normalized class $\mathfrak{A}(R, S)$ have precisely $u$ rows and $v$ columns composed entirely of 1 's and let $\tilde{\sigma} \neq \max (u, v)$. Then there exists a matrix $A_{\tilde{\sigma}}$ of trace $\tilde{\sigma}$ in $\mathfrak{A}(R, S)$ of the form

$$
A_{\tilde{\sigma}}=\left[\begin{array}{lll}
J & J_{1} & * \\
J_{2} & \tilde{J} & * \\
* & * & 0
\end{array}\right] .
$$

Here $J$ is a matrix of 1 's of order $\tilde{\sigma} . J_{1}$ of size $\tilde{\sigma}$ by $s$ and $J_{2}$ of size $t$ by $\tilde{\sigma}$ are matrices of 1 's. $\tilde{J}$ is a matrix with 0 's in the main diagonal positions of $A_{\tilde{\sigma}}$ and 1 's in all other positions. 0 is a zero matrix. (The cases $s=0$ and $t=0$ are not excluded.)

The canonical forms (3.2) and (3.4) yield explicit formulas for $\bar{\sigma}$ and $\tilde{\sigma}$ in terms of the elements of the structure matrix $T=\left[t_{e f}\right]$ of the normalized class $\mathfrak{A}(R, S)$ [62]. 
THEOREM 3.3. Let $\bar{\sigma}$ be the maximal and $\tilde{\sigma}$ the minimal trace of the matrices in the normalized class $\mathfrak{A}(R, S)$. Then

$$
\bar{\sigma}=\min _{e, f}\left\{t_{e f}+\max (e, f)\right\} \quad(e=0,1, \cdots, m ; f=0,1, \cdots, n)
$$

and

$$
\tilde{\sigma}=\max _{e, f}\left\{\min (e, f)-t_{e f}\right\} \quad(e=0,1, \cdots, m ; f=0,1, \cdots, n)
$$

It is easy to verify that $\tilde{\sigma}=\bar{\sigma}$ if and only if the structure matrix contains a zero on the main diagonal. The problem of intermediate values

$$
\tilde{\sigma} \leqq \sigma \leqq \bar{\sigma}
$$

is settled by the following theorem [62].

THEOREM 3.4. The traces of the matrices in the normalized class $\mathfrak{A}(R, S)$ take on all intermediate values in the interval (3.7) unless $\mathfrak{A}(R, S)$ contains a matrix of the form

$$
A=\left[\begin{array}{lll}
J & J^{*} & * \\
J^{* T} & I_{c} & 0 \\
* & 0 & 0
\end{array}\right] .
$$

Here $J$ is a matrix of 1's of order $e, J^{*}$ is a rectangular matrix of 1 's, $J^{* T}$ is the transpose of $J^{*}, I_{c}$ is the identity matrix or the complement of this matrix, and the 0's are zero matrices. The order of $I_{c}$ is $g$ with $g \geqq 2$. (The cases $e=0, e+g=m$, and $e+g=n$ are not excluded.)

The normality assumption for the class $\mathfrak{A}(R, S)$ is a genuine restriction in our study of the maximal and minimal trace. This assumption appears to be essential in order to obtain the simple formulas (3.5) and (3.6). But more insight into the behavior of the trace in an arbitrary $\mathfrak{H}(R, S)$ would certainly be valuable. Fulkerson has investigated feasibility conditions for the existence of a $(0,1)$-matrix of order $n$ with specified row and column sums and zero trace [14]. This approach utilizes the theory of network flows $[11 ; 12 ; 13]$. The criterion assumes an especially simple form under the normality assumption. Indeed, formulas (3.5) and (3.6) may be derived by network flows.

4. Term ranks. Let $A$ be a $(0,1)$-matrix of size $m$ by $n$. The term rank $\rho$ of $A$ is the maximal trace obtained from $A$ under arbitrary permutations of the rows and of the columns of $A$. Thus the term rank of $A$ equals the maximal number of 1 's that may be chosen in 
$A$ with no two in the same row or column. The classical theorem on the subject asserts that the term rank of $A$ is also equal to the minimal number of rows and columns that contain all of the 1 's in $A$ [40]. Let $A$ be the incidence matrix for the subsets $X_{1}, X_{2}, \cdots, X_{m}$ of the set $X$ of $n$ elements $x_{1}, x_{2}, \cdots, x_{n}$. The term rank of $A$ is invariant under permutations of rows and columns of $A$. Consequently the term rank is independent of the particular labelling of elements $x_{1}, x_{2}, \cdots, x_{n}$ and subsets $X_{1}, X_{2}, \cdots, X_{m}$ of $X$. In fact the term rank equals the maximal integer $\rho$ for which there exists a labelling of elements $x_{1}^{\prime}, x_{2}^{\prime}, \cdots, x_{n}^{\prime}$ and subsets $X_{1}^{\prime}, X_{2}^{\prime}, \cdots, X_{m}^{\prime}$ such that $x_{i}^{\prime}$ belongs to $X_{i}^{\prime}$ for $i=1,2, \cdots, \rho$. The special case in which $\rho=m$ is of considerable importance in its own right. Then the subsets $X_{1}, X_{2}, \cdots, X_{m}$ of $X$ are said to possess a system of distinct representatives. The topics described thus far in this section are subject to many generalizations and refinements. A very extensive literature is available. See, for example, $[30 ; 34 ; 42]$.

Now let $\bar{\rho}$ equal the maximal and $\tilde{\rho}$ the minimal term rank of the matrices $A$ in the normalized class $\mathfrak{A}(R, S)$. Let $\rho$ be an arbitrary integer in the interval

$$
\tilde{\rho} \leqq \rho \leqq \bar{\rho} .
$$

The interchange theorem implies that there exists a matrix $A_{\rho}$ in the normalized class $\mathfrak{A}(R, S)$ of term rank $\rho$. The reason for this is that a single interchange may alter the term rank of a matrix by at most 1. We remark that an interchange may alter the trace of a matrix by 2 and this accounts for the complication in intermediate traces described in Theorem 3.4.

The integer $\bar{\rho}$ has been investigated in detail in [59] and the main content of this paper may be summarized in the following theorem.

Theorem 4.1. Let $A$ be a matrix in the normalized class $\mathfrak{A}(R, S)$ and let $\bar{\rho}<m, n$. Then

$$
A=\left[\begin{array}{cc}
W & X \\
Y & Z
\end{array}\right] .
$$

Here $W$ is of specified size e by $f(0<e<m ; 0<f<n)$ and

$$
N_{0}(W)+N_{1}(Z)=\bar{\rho}-(e+f) .
$$

There exists a matrix $A_{\bar{\rho}}$ of term rank $\bar{\rho}$ in $\mathfrak{A}(R, S)$ such that

$$
N_{0}(W)=0
$$




$$
N_{1}(Z)=\bar{\rho}-(e+f) .
$$

We make no attempt to prove Theorem 4.1 here. An easy consequence of this theorem is a striking formula for $\bar{\rho}$ in terms of the elements of the structure matrix $T=\left[t_{e f}\right][59 ; 62]$.

TheOREM 4.2. The maximal term rank $\bar{\rho}$ for the matrices in the normalized class $\mathfrak{A}(R, S)$ is given by

$$
\bar{\rho}=\min _{e, f}\left\{t_{e f}+(e+f)\right\} \quad(e=0,1, \cdots, m ; f=0,1, \cdots, n) .
$$

A network flow derivation of formula (4.6) would be of interest. Attempts have been made in this direction but they have been unsuccessful up to now.

Theorem 4.3. Let the normalized class $\mathfrak{A}(R, S)$ be without invariant 1 's and let $\bar{\rho}<m, n$. Then $\tilde{\rho}<\bar{\rho}$.

Theorem 4.3 is also an easy consequence of Theorem 4.1. But note that Theorem 4.3 need not be valid if the hypothesis on invariant 1 's is deleted. The class consisting of a maximal matrix $\bar{A}$ has $\tilde{\rho}=\bar{\rho}$. Nor may the restriction $\bar{\rho}<m, n$ be removed. For the class of permutation matrices has $\tilde{\rho}=\bar{\rho}$. An unsettled problem of interest calls for a neat classification of all classes $\mathfrak{A}(R, S)$ with $\tilde{\rho}=\bar{\rho}$.

The integer $\tilde{\rho}$ is elusive and difficult to handle. However, Haber has devised an effective algorithm for the evaluation of $\tilde{\rho}$ [19]. A simple formula for $\tilde{\rho}$ analogous to (4.6) for $\bar{\rho}$ may not exist, but $\tilde{\rho}$ deserves further study in this connection.

5. Widths and heights. We describe next the concepts of widths and heights introduced in [15]. Let $A$ be a matrix in the normalized class $\mathfrak{H}(R, S)$. Let $\alpha$ be an integer in the interval

$$
1 \leqq \alpha \leqq r_{m}
$$

and let $\epsilon$ be an integer in the interval

$$
1 \leqq \epsilon \leqq n .
$$

Suppose that $A$ has an $m$ by $\epsilon$ submatrix $E^{*}$ each of whose row sums is at least $\alpha$. Then the $\epsilon$ columns of $E^{*}$ form an $\alpha$-set of representatives for the matrix $A$. Let $\epsilon(\alpha)$ be the minimal number of columns of $A$ that form an $\alpha$-set of representatives for $A$. Such a column set is called a minimal $\alpha$-set of representatives for $A$ and $\epsilon(\alpha)$ is called the $\alpha$-width of $A$. The integer $\alpha$ and the matrix $A$ uniquely determine $\epsilon(\alpha)$. Note that $\epsilon(\alpha)$ is invariant under arbitrary permutations of the rows and the columns of $A$. However, the $\alpha$-width of $A^{T}$, the transpose of 
$A$, may differ decidedly from that of $A$. This is not the case for trace and term rank, both of which remain invariant under transposition.

Now let $E^{*}$ be a submatrix of $A$ of size $m$ by $\epsilon(\alpha)$ that yields a minimal $\alpha$-set of representatives for $A$. Let $E$ be the submatrix of $E^{*}$ composed of all rows of $E^{*}$ that contain $\alpha$ 1's and $\epsilon(\alpha)-\alpha 0$ 's. $E$ is called a critical $\alpha$-submatrix of $A$. It follows without difficulty that $A$ has an $\alpha$-width $\epsilon(\alpha)$ for each $\alpha$ in the interval $1 \leqq \alpha \leqq r_{m}$. $A$ critical $\alpha$-submatrix $E$ of $A$ associated with an $\alpha$-width $\epsilon(\alpha)$ cannot be empty and contains no zero columns.

Each critical $\alpha$-submatrix $E$ of $A$ contains $\epsilon(\alpha)$ columns. But the number of rows in the various critical $\alpha$-submatrices of $A$ need not be fixed. Let $E$ be a critical $\alpha$-submatrix containing the minimal number of rows $\delta(\alpha)$. The positive integer $\delta(\alpha)$ is called the $\alpha$-height of $A$. It is clear that

$$
\epsilon(1)<\epsilon(2)<\cdots<\epsilon\left(r_{m}\right)
$$

and

$$
\delta(1) \geqq \epsilon(1) .
$$

The width and the height of a $(0,1)$-matrix have an important set theoretic interpretation. Let $A$ be the incidence matrix for the subsets $X_{1}, X_{2}, \cdots, X_{m}$ of $X$. No loss is entailed by taking $A$ in the normalized class $\mathfrak{A}(R, S)$. A minimal $\alpha$-set of representatives for $A$ yields a subset $X^{*}$ of $\epsilon(\alpha)$ elements of $X$. The subset $X^{*}$ has the property that each $X_{i} \cap X^{*}$ contains at least $\alpha$ elements $(i=1,2, \cdots, m)$. No subset of $X$ containing fewer than $\epsilon(\alpha)$ elements possesses this property. At least $\delta(\alpha)$ of the sets $X_{i} \cap X^{*}$ contain exactly $\alpha$ elements. If $\alpha=1$, then $X^{*}$ has the property that each $X_{i} \cap X^{*}$ is nonempty and no subset of $X$ containing fewer than $\epsilon(1)$ elements possesses this property. We mention in passing that the 1 -width of a matrix also arises naturally in certain network problems. Consider the problem of finding the fewest number of nodes in a network that touch all links of the network. This is equivalent to finding the 1-width of the incidence matrix of links versus nodes. The well-known "eight queens" chessboard problem may be reduced to a problem of this type [16].

Let $1 \leqq \alpha \leqq r_{m}$. Then each $A$ in the normalized class $\mathfrak{A}(R, S)$ determines an $\alpha$-width $\epsilon(\alpha)$. For each $\alpha$ let the minimal of these $\epsilon(\alpha)$ 's over all $A$ in $\mathfrak{A}(R, S)$ be denoted by

$$
\tilde{\boldsymbol{\epsilon}}=\tilde{\boldsymbol{\epsilon}}(\alpha) .
$$

We call $\tilde{\boldsymbol{\epsilon}}=\tilde{\boldsymbol{\epsilon}}(\alpha)$ the minimal $\alpha$-width of the class $\mathfrak{A}(R, S)$. Consider 
now all matrices $A_{\tilde{\epsilon}}$ in $\mathfrak{A}(R, S)$ of $\alpha$-width $\tilde{\boldsymbol{\epsilon}}(\alpha)$. Each of these matrices has an $\alpha$-height $\delta(\alpha)$. Let

$$
\tilde{\delta}=\tilde{\delta}(\alpha)
$$

be the minimal of the $\delta(\alpha)$ 's over the matrices $A_{\tilde{\epsilon}}$ in $\mathfrak{A}(R, S)$ of $\alpha$-width $\tilde{\boldsymbol{\epsilon}}(\alpha)$. We call $\tilde{\delta}=\tilde{\delta}(\alpha)$ the multiplicity of $\alpha$ with respect to $\tilde{\boldsymbol{\epsilon}}(\alpha)$. It is clear that

$$
\tilde{\boldsymbol{\epsilon}}(1)<\tilde{\boldsymbol{\epsilon}}(2)<\cdots<\tilde{\boldsymbol{\epsilon}}\left(r_{m}\right)
$$

and

$$
\tilde{\delta}(1) \geqq \tilde{\epsilon}(1) .
$$

Similarly for each $\alpha$ let the maximum of the $\epsilon(\alpha)$ 's over all $A$ in $\mathfrak{A}(R, S)$ be denoted by

$$
\bar{\epsilon}=\bar{\epsilon}(\alpha) \text {. }
$$

We call $\bar{\epsilon}=\bar{\epsilon}(\alpha)$ the maximal $\alpha$-width of the class $\mathfrak{A}(R, S)$. A direct application of the interchange theorem allows us to prove that if $\epsilon$ is an integer in the interval

$$
\tilde{\epsilon} \leqq \epsilon \leqq \bar{\epsilon},
$$

then there exists a matrix $A_{\epsilon}$ in $\mathfrak{Y}(R, S)$ of $\alpha$-width $\epsilon$.

The following theorem yields a matrix $A_{\tilde{\boldsymbol{\epsilon}}}$ in $\mathfrak{Y}(R, S)$ of $\alpha$-width $\tilde{\boldsymbol{\epsilon}}$ and with an unusually simple block decomposition. The canonical form (5.11) may be obtained by applying interchanges that utilize the minimal properties of $\tilde{\epsilon}$ and $\tilde{\delta}[15]$.

TheOREM 5.1. Let $\tilde{\boldsymbol{\epsilon}}=\tilde{\boldsymbol{\epsilon}}(\alpha)$ be the minimal $\alpha$-width of the normalized class $\mathfrak{A}(R, S)$. Let $\tilde{\delta}=\tilde{\delta}(\alpha)$ be the multiplicity of $\alpha$ with respect to $\tilde{\epsilon}(\alpha)$. Then there exists a matrix $A_{\tilde{\epsilon}}$ of $\alpha$-width $\tilde{\epsilon}$ in $\mathfrak{A}(R, S)$ of the form

$$
A_{\tilde{\boldsymbol{\epsilon}}}=\left[\begin{array}{c|c|c}
M & J & * \\
\hline F & & \\
\hline E & * & 0
\end{array}\right] .
$$

Here $E$ is a critical $\alpha$-submatrix of $A_{\tilde{\epsilon}}$ of size $\tilde{\delta}$ by $\tilde{\epsilon} . M$ is a matrix of size $e$ by $\tilde{\epsilon}$ with $\alpha+1$ or more 1's in each row. $F$ is a matrix of size $m-(e+\tilde{\delta})$ by $\tilde{\epsilon}$ with exactly $\alpha+1$ 1's in each row. $J$ is a matrix of size $e$ by $f-\tilde{\epsilon}$ consisting entirely of 1's, and 0 is a zero matrix. Each of the first $\tilde{\boldsymbol{\epsilon}}$ columns of $A_{\tilde{\boldsymbol{\epsilon}}}$ contains more than $m-\tilde{\delta} 1$ 's. The degenerate cases $e=0, e+\tilde{\delta}=m, f=\tilde{\epsilon}$, and $f=n$ are not excluded. 
We now define invariants $N(\epsilon, e, f)$ of $\mathfrak{A}(R, S)$ that are generalizations of the invariants $t_{e f}$ of (2.4). These invariants are effective in yielding explicit formulas for $\tilde{\boldsymbol{\epsilon}}(\alpha)$ and $\tilde{\delta}(\alpha)$. Let

$$
N(\epsilon, e, f)=t_{e f}+\left(s_{1}+s_{2}+\cdots+s_{\epsilon}\right)-e \epsilon .
$$

Here $\epsilon, e, f$ are integer parameters such that

$$
\begin{aligned}
& 0 \leqq \epsilon \leqq n, \\
& 0 \leqq e \leqq m, \\
& \epsilon \leqq f \leqq n .
\end{aligned}
$$

Note that

$$
N(0, e, f)=t_{e f} \quad(e=0,1, \cdots, m ; f=0,1, \cdots, n) .
$$

Let $A$ be in the normalized class $\mathfrak{A}(R, S)$ and write

$$
A=\left[\begin{array}{lll}
* & Y & * \\
X & * & Z
\end{array}\right]
$$

where $X$ is of size $m-e$ by $\epsilon$ and $Y$ is of size $e$ by $f-\epsilon$. Then it is easy to verify that

$$
N(\epsilon, e, f)=N_{1}(X)+N_{0}(Y)+N_{1}(Z) .
$$

THEOREM 5.2. The minimal $\alpha$-width $\tilde{\epsilon}(\alpha)$ of the normalized class $\mathfrak{U}(R, S)$ equals the first nonnegative integer $\epsilon$ such that

$$
N(\epsilon, e, f) \geqq \alpha(m-e),
$$

for all integer parameters $e$ and $f$ restricted by $0 \leqq e \leqq m$ and $\epsilon \leqq f \leqq n$. Let

$$
\tilde{\gamma}=\min _{e, f}\{N(\tilde{\epsilon}-1, e, f)+\alpha e\},
$$

where $0 \leqq e \leqq m$ and $\tilde{\boldsymbol{\epsilon}}-1 \leqq f \leqq n$. Then

$$
\tilde{\delta}(\alpha)=(\alpha+1) m-\tilde{\gamma}-s_{\tilde{\epsilon}} .
$$

Theorem 5.1 plays a critical role in the derivation of Theorem 5.2 [15]. Actually formula (5.19) is a rather direct consequence of Theorem 5.1 once the appropriate formula has been guessed. It should be remarked that (5.19) was obtained initially from a study of network flows.

A formula for $\bar{\epsilon}(\alpha)$ analogous to (5.19) for $\tilde{\epsilon}(\alpha)$ does not exist. In fact we will show subsequently that for certain important classes $\mathfrak{H}(R, S)$ the value of $\bar{\epsilon}(1)$ is determined by deep arithmetical properties of the class. A reasonable computational procedure for $\bar{\epsilon}(\alpha)$ would be in itself of the utmost value. 
6. The class $\mathfrak{A}(K, K)$. Thus far we have associated with the $(0,1)$ matrix $A$ in the normalized class $\mathfrak{U}(R, S)$ various integers that give us insight into the combinatorial structure of $A$. The possibilities have been by no means exhausted. For example, let $\tilde{\pi}$ be the minimal and $\bar{\pi}$ the maximal ranks of the matrices in $\mathfrak{A}(R, S)$. If $\pi$ is an arbitrary integer in the interval

$$
\tilde{\pi} \leqq \pi \leqq \bar{\pi},
$$

then by the interchange theorem there exists a matrix in $\mathfrak{A}(R, S)$ of rank $\pi$. But at the present time almost nothing is known about $\tilde{\pi}$ and $\bar{\pi}$. Or consider the symmetric matrices $B_{1}$ and $B_{2}$ defined by

$$
A A^{T}=B_{1}, \quad A^{T} A=B_{2},
$$

where $A^{T}$ denotes the transpose of $A$. Nontrivial information on $B_{1}$ and $B_{2}$ as $A$ ranges over $\mathfrak{A}(R, S)$ would be very valuable. But deep theorems along these lines may well be far beyond the present range of our knowledge.

For the remainder of the discussion we specialize $\mathfrak{A}(R, S)$ to the class $\mathfrak{A}(K, K)$ defined by

$$
m=n=v
$$

and

$$
R=S=K=(k, k, \cdots, k),
$$

where $k$ is a fixed integer in the interval

$$
1 \leqq k \leqq v .
$$

Thus $\mathfrak{A}(K, K)$ consists of all $(0,1)$-matrices of order $v$ with exactly $k$ 1 's in each row and column. We call the matrices of these $v$ classes the $(0,1)$-doubly stochastic matrices. For $k=1, \mathfrak{A}(K, K)$ consists of the $v$ ! permutation matrices of order $v$ and for $k=v, \mathfrak{A}(K, K)$ consists of the matrix $J$ of order $v$.

For the class $\mathfrak{A}(K, K)$, it is trivial to verify that

$$
\begin{aligned}
& \tilde{\sigma}=0, \quad \bar{\sigma}=v, \\
& \tilde{\sigma}=\bar{\sigma}=v,
\end{aligned}
$$$$
(1 \leqq k<v) \text {, }
$$$$
(k=v) \text {. }
$$

A well-known theorem on term rank [40] asserts that for the class $\mathfrak{U}(K, K)$

$$
\tilde{\rho}=\bar{\rho}=v .
$$

This implies that for an arbitrary matrix $A$ in $\mathfrak{A}(K, K)$

$$
A=P_{1}+P_{2}+\cdots+P_{k},
$$


where the $P_{i}$ are permutation matrices. The results on minimal widths simplify considerably for the case of the class $\mathfrak{A}(K, K)$ [15]. For this class $\tilde{\epsilon}(\alpha)$ is the first integer $\epsilon$ such that

$$
\epsilon \geqq \alpha v / k
$$

and

$$
\tilde{\delta}(\alpha)=(\alpha+1) v-k \tilde{\epsilon}(\alpha) .
$$

It is clear that much of the material discussed in the preceding sections becomes almost trivial for the class $\mathfrak{A}(K, K)$. But $\mathfrak{A}(K, K)$ possesses deep and fascinating problems in its own right. Many of these problems involve combinatorial designs in one form or another and are discussed in the concluding sections.

7. Combinatorial designs. Let $v$ elements $x_{1}, x_{2}, \cdots, x_{v}$ be arranged into $v$ sets $X_{1}, X_{2}, \cdots, X_{v}$ such that every set contains exactly $k$ distinct elements and such that every pair of sets has exactly $\lambda$ elements in common $(0<\lambda<k<v)$. Such a configuration is called a $v, k, \lambda$ configuration. Let $a_{i j}=1$ if $x_{j}$ is in set $X_{i}$, and 0 otherwise. The matrix $A=\left[a_{i j}\right]$ of order $v$ is called the incidence matrix of the $v, k, \lambda$ configuration. Let $v, k$, and $\lambda$ be integers such that $0<\lambda<k<v$. Then it is clear that a $v, k, \lambda$ configuration exists if and only if there exists a $(0,1)$-matrix $A$ of order $v$ such that

$$
A A^{T}=B,
$$

where $A^{T}$ denotes the transpose of $A$ and where $B$ has $k$ in the main diagonal and $\lambda$ in all other positions. For a $v, k, \lambda$ configuration, one may prove that

$$
\lambda=k(k-1) /(v-1) .
$$

Moreover, the incidence matrix of a $v, k, \lambda$ configuration is normal, that is

$$
A A^{T}=A^{T} A .
$$

The derivations of $(7.2)$ and $(7.3)$ are not difficult $[53 ; 56 ; 63]$.

The central problem in the study of these configurations is the determination of the precise range of values of $v, k$, and $\lambda$ for which configurations exist. Formula (7.2) gives a first necessary condition and in discussing these configurations it is assumed that the integer parameters $v, k$, and $\lambda$ satisfy this requirement.

Theorem 7.1. Suppose that $a v, k, \lambda$ configuration exists. If $v$ is even, then $k-\lambda$ equals a square. If $v$ is odd, then the Diophantine equation 


$$
x^{2}=(k-\lambda) y^{2}+(-1)^{(v-1) / 2} \lambda z^{2}
$$

possesses a nonzero integral solution.

Theorem 7.1 appears in [6]. The case of $v$ even follows easily upon taking determinants in the matric equation $A A^{T}=B$. The case of $v$ odd is far from trivial but may be derived by methods which are entirely elementary. Theorem 7.1 accounts for all known excluded configurations so that one may conjecture their existence in all other cases. An extensive literature centers around Theorem 7.1 and the matric equations (7.1) and (7.3). Some key references are listed in the bibliography. Surveys with more extensive references are also available $[56 ; 63]$.

Certain specializations of the values of $v, k$, and $\lambda$ lead to classical configurations. The configuration with parameters $v=N^{2}+N+1$, $k=N+1, \lambda=1, N \geqq 2$ is equivalent to a finite projective plane $\pi$ of order $N$. Mention should be made of the Bruck-Ryser theorem on the nonexistence of these planes [5].

THEOREM 7.2. Let $N \equiv 1$ or $2(\bmod 4)$ and let the squarefree part of $N$ contain at least one prime factor $\equiv 3(\bmod 4)$. Then $\pi$ of order $N$ does not exist.

The original proof of Theorem 7.2 utilized the Minkowski-Hasse invariants of a quadratic form [5]. These invariants have subsequently been used in a wide variety of combinatorial investigations. It is easy to verify that Theorem 7.1 with $\lambda=1$ reduces to Theorem 7.2. The first projective plane for which the existence problem is undecided has order $N=10$. The associated $v, k, \lambda$ configuration has parameters $v=111, k=11, \lambda=1$.

Another important specialization of values of $v, k$, and $\lambda$ is $v=4 t-1$, $k=2 t, \lambda=t$. Such a $v, k, \lambda$ configuration is equivalent to a Hadamard matrix of order $4 t$. These are the matrices with entries \pm 1 and of order $h$ such that

$$
H H^{T}=h I .
$$

Here $H^{T}$ is the transpose of $H$ and $I$ is the identity matrix of order $h$. If $H$ exists, then it is easy to verify that the order of $H$ is 1,2 , or $\equiv 0(\bmod 4)$. The existence of Hadamard matrices for orders 1 and 2 is trivial, and it is conjectured that they exist for all orders $\equiv 0(\bmod 4)$. The first undecided order is $h=92$, and this corresponds to the configuration $v=91, k=46, \lambda=23$.

8. Concluding remarks. We return now to the class $\mathfrak{A}(K, K)$ of all $(0,1)$-matrices of order $v$ with exactly $k 1$ 's in each row and column, 
where $k$ is a fixed integer in the interval $1 \leqq k \leqq v$. We have already remarked that for the class $\mathfrak{A}(K, K)$

$$
\tilde{\rho}=\bar{\rho}=v .
$$

Thus all matrices in $\mathfrak{A}(K, K)$ have the same term rank $v$. But suppose that for an $A$ in $\mathfrak{A}(K, K)$ we ask for the number of distinct ways in which this term rank is attained. This leads us to the concept of the permanent of a matrix.

Let $A=\left[a_{i j}\right]$ be an $m$ by $n$ matrix with the $a_{i j}$ real and $m \leqq n$. Then the permanent of $A$ is defined by

$$
\operatorname{per}(A)=\sum a_{1 i_{1}} a_{2 i_{2}} \cdots a_{m i_{m}},
$$

where $i_{1}, i_{2}, \cdots, i_{m}$ range over all permutations of $m$ integers chosen from among $1,2, \cdots, n$. It is clear that if $A$ is a $(0,1)$-matrix, then per $(A)>0$ if and only if the term rank $\rho$ of $A$ is $m$. Moreover, if $A$ is an incidence matrix for the $m$ subsets $X_{1}, X_{2}, \cdots, X_{m}$ of $X$, then these subsets possess per $(A)$ systems of distinct representatives. In the case of a square matrix it is natural to compare per $(A)$ with the determinant $\operatorname{det}(A)$. But these two functions of $A$ bear only a superficial resemblance to one another. It is usually much more difficult to evaluate the permanent. Also the permanent possesses a number of combinatorial characteristics not shared by the determinant. For example, let $J$ be the matrix of 1 's of order $v$ and let $I$ be the identity matrix of order $v$. Then

$$
\operatorname{per}(J)=v !
$$

and

$$
\operatorname{per}(J-I)=v !\left(1-\frac{1}{1 !}+\frac{1}{2 !}-\cdots+(-1)^{v} \frac{1}{v !}\right) .
$$

Each matrix $A$ in the class $\mathfrak{A}(K, K)$ has per $(A)>0$. But little is known about the minimal value of per $(A)$ for $A$ in $\mathfrak{A}(K, K)$. If $\mathfrak{A}(K, K)$ contains an incidence matrix of a $v, k, \lambda$ configuration, then very limited empirical information suggests that the permanent of this matrix is small or even minimal in $\mathfrak{A}(K, K)$. Computation becomes prohibitive in situations of this type even for very small values of $v$. Let $J$ denote the matrix of 1 's of order $k$. Let $k$ divide $v$ and let $J^{*}$ denote the direct sum of $v / k$ matrices $J$. Then $J^{*}$ belongs to $\mathfrak{Y}(K, K)$ and we conjecture that in this case the permanent of $J^{*}$ is maximal in $\mathfrak{Y}(K, K)$. This suggests that the permanent of a matrix $A$ in $\mathfrak{A}(K, K)$ is related to the $v(v-1) / 2$ integers above the main diagonal in $A A^{T}$. These integers lie in the interval $0 \leqq x \leqq k$. For in- 
cidence matrices of $v, k, \lambda$ configurations, each of the $v(v-1) / 2$ integers is equal to $\lambda$. For the direct sum matrix $J^{*}, v(k-1) / 2$ of the integers are equal to $k$ and the remaining integers are equal to 0 .

The preceding remarks are motivated in part by an analogous situation involving doubly stochastic matrices. A matrix $X$ of order $v$ is doubly stochastic provided its entries are nonnegative reals and its row and column sums are each equal to 1 . It is trivial to verify that if $X$ is doubly stochastic, then per $(X) \leqq 1$, with equality if and only if $X$ is a permutation matrix. In 1926 van der Waerden suggested the problem of determining the minimum of per $(X)$ for $X$ doubly stochastic [71]. This problem is still unsolved. But recently Marcus and Newman [44] have made advances toward a solution. The present conjecture is that for $X$ doubly stochastic of order $v$, per $(X)$ $\geqq v ! / v^{v}$, with equality if and only if $X$ is the doubly stochastic matrix all of whose entries are $1 / v$.

Very little is known about the permanent of the incidence matrix of a $v, k, \lambda$ configuration. A formula for the permanent is not available even if it is assumed that the incidence matrix of the configuration is cyclic. Nikolai has used electronic computers to carry out extensive calculations in this area [47]. For projective planes of orders 2,3 , and 4 , the permanents are $24,3,852$, and $18,534,400$, respectively. The permanent is invariant under permutations of rows and columns and under transposition. Let $A$ and $A^{\prime}$ be two $v, k, \lambda$ incidence matrices not transformable into one another by these operations. We conjecture that these matrices possess distinct permanents. Their determinants are, of course, equal in absolute value.

Let $Z$ denote an incidence matrix of a projective plane of order 2 . The matrix $Z$ is of order 7 and has the striking property

$$
\text { per }(Z)=\text { abs. val. } \operatorname{det}(Z)=24 \text {. }
$$

Tinsley has utilized this observation to carry out some interesting investigations involving permanents [68]. The following is one of his central theorems.

Theorem 8.1. Let $A$ be a cyclic matrix in $\mathfrak{U}(K, K)$. If $k>3$, then

$$
\text { per }(A)>\text { abs. val. } \operatorname{det}(A) \text {. }
$$

For $k=3$, if

$$
\operatorname{per}(A)=\text { abs. val. } \operatorname{det}(A),
$$

then upon permutations of rows and columns, $A$ becomes the direct sum of the matrix $Z$ taken e times. Hence $v=7$ e and per $(A)=(24)^{e}$. 
Theorem 8.1 appears to be valid without the restriction that $A$ be cyclic. But this is an unsettled question at the present time.

Incidence matrices of $v, k, \lambda$ configurations also arise in extremal investigations of a rather different type. The following theorem is a byproduct of a study of inequalities of compound and induced matrices $[60 ; 61]$.

TheOREM 8.2. Let $Q$ be a $(0,1)$-matrix of order $v$ and let $Q$ contain exactly $\tau$ 1's. Define $k$ and $\lambda$ by

$$
\begin{aligned}
\tau & =k v, \\
\lambda & =k(k-1) /(v-1),
\end{aligned}
$$

and suppose that $0<\lambda<k<v$. Let $\alpha_{1}, \alpha_{2}, \cdots, \alpha_{v}$ denote the $v$ characteristic roots of $Q Q^{T}$. Let $\Sigma_{r}$ be the rth elementary symmetric function of $\alpha_{1}, \alpha_{2}, \cdots, \alpha_{v}$ and let $\Sigma_{r}^{*}$ be the rth homogeneous product sum of $\alpha_{1}, \alpha_{2}, \cdots, \alpha_{v}$ (i.e. $\left.\Sigma_{r}^{*}=\alpha_{1}^{r}+\alpha_{1}^{r-1} \alpha_{2}+\cdots\right)$. Then

$$
\Sigma_{r} \leqq\left(\begin{array}{c}
v \\
r
\end{array}\right)(k+(r-1) \lambda)(k-\lambda)^{r-1} \quad(r=1,2, \cdots, v),
$$

and

$$
\begin{aligned}
& \Sigma_{r}^{*} \geqq \sum_{i=0}^{r}\left(\begin{array}{c}
v+i-2 \\
i
\end{array}\right)(k+(v-1) \lambda)^{r-i}(k-\lambda)^{i} \\
&(r=1,2, \cdots) .
\end{aligned}
$$

Equality holds for $r=1$. If equality holds in either (8.10) or (8.11) for an $r>1$, then $Q$ is the incidence matrix of $a v, k, \lambda$ configuration and equality holds throughout (8.10) and (8.11) for all $r$.

It seems unlikely that inequalities of the type (8.10) and (8.11) will settle some of the deep arithmetrical problems associated with $v, k, \lambda$ configurations. However, such inequalities are of interest in their own right. Note that (8.10) implies

$$
(\operatorname{det}(Q))^{2} \leqq k^{2}(k-\lambda)^{v-1},
$$

where equality holds if and only if $Q$ is the incidence matrix of a $v, k, \lambda$ configuration [57]. Thus if the class $\mathfrak{A}(K, K)$ contains an incidence matrix of a $v, k, \lambda$ configuration, then the determinant of this matrix is maximal in absolute value over the matrices of the class. This is in a sense surprising because our earlier remarks implied that the permanent of such a matrix may be minimal in the class. 
We conclude with some observations on $\alpha$-widths in the class $\mathfrak{U}(K, K)$. We have already noted that the minimal $\alpha$-width $\tilde{\epsilon}(\alpha)$ of the class $\mathfrak{A}(K, K)$ is the first integer $\epsilon$ such that

$$
\epsilon \geqq \alpha v / k \text {. }
$$

One may attempt to find a formula for the maximal $\alpha$-width $\bar{\epsilon}(\alpha)$ of the class $\mathfrak{A}(K, K)$. But the following remarks point out the difficulty of such an undertaking. With the class $\mathfrak{A}(K, K)$ we associate the complementary class $\mathfrak{A}\left(K^{\prime}, K^{\prime}\right)$ of all $(0,1)$-matrices of order $v$ with exactly $k^{\prime}=v-k 1$ 's in each row and column.

TheOREm 8.3. Let $\mathfrak{A}(K, K)$ be the class with parameters $v=N^{2}+N$ $+1, k=N+1, N \geqq 2$ and let $\mathfrak{H}\left(K^{\prime}, K^{\prime}\right)$ be the complementary class with parameters $v=N^{2}+N+1, k^{\prime}=N^{2}, N \geqq 2$. If a projective plane $\pi$ of order $N$ exists, then the maximal 1-width of the class $\mathfrak{A}\left(K^{\prime}, K^{\prime}\right)$ satisfies

$$
\bar{\epsilon}(1)=3 \text {. }
$$

If a projective plane $\pi$ of order $N$ does not exist, then the maximal 1-width of the class $\mathfrak{U}\left(K^{\prime}, K^{\prime}\right)$ satisfies

$$
\bar{\epsilon}(1)=2 .
$$

The proof is almost immediate. For the class $\mathfrak{A}(K, K)$ has parameters $v=N^{2}+N+1, k=N+1, N \geqq 2$ and the complementary class $\mathfrak{U}\left(K^{\prime}, K^{\prime}\right)$ has parameters $v=N^{2}+N+1, k^{\prime}=N^{2}, N \geqq 2$. The average value of the inner product of two distinct column vectors of a matrix in $\mathfrak{P}\left(K^{\prime}, K^{\prime}\right)$ is

$$
\lambda^{\prime}=\frac{N^{2}\left(N^{2}-1\right)}{N^{2}+N}=N(N-1) .
$$

Now let $A^{\prime}$ be a matrix in $\mathfrak{A}\left(K^{\prime}, K^{\prime}\right)$ such that all inner products of its distinct column vectors equal $\lambda^{\prime}=N(N-1)$. Then the 1 -width of $A^{\prime}$ is $\epsilon(1)=3$. Let $A^{*}$ be a matrix in $\mathfrak{A}\left(K^{\prime}, K^{\prime}\right)$ that violates the inner product requirement of $A^{\prime}$. Then $A^{*}$ has a pair of column vectors whose inner product is less than the average value $\lambda^{\prime}=N(N-1)$. But since the order of $A^{*}$ is $N^{2}+N+1$, it follows that the 1 -width of $A^{*}$ is $\epsilon(1)=2$. Hence if $A^{\prime}$ exists in $\mathfrak{A}\left(K^{\prime}, K^{\prime}\right)$, then $\bar{\epsilon}(1)=3$ and if $A^{\prime}$ does not exist in $\mathfrak{U}\left(K^{\prime}, K^{\prime}\right)$, then $\bar{\epsilon}(1)=2$. But the existence of $A^{\prime}$ in the class $\mathfrak{H}\left(K^{\prime}, K^{\prime}\right)$ is precisely equivalent to the existence of an incidence matrix $A$ of a projective plane $\pi$ of order $N$ in the class $\mathfrak{A}(K, K)$. This proves the assertion of the theorem. The interconnection between $\alpha$-widths and combinatorial designs is a topic that deserves careful study. 


\section{BibliogRAPHY}

1. A. A. Albert, Rational normal matrices satisfying the incidence equation, Proc. Amer. Math. Soc. vol. 4 (1953) pp. 554-559.

2. R. C. Bose, On the construction of balanced incomplete block designs, Ann. Eugenics vol. 9 (1939) pp. 353-399.

3. R. C. Bose and Dale M. Mesner, On linear associative algebras corresponding to association schemes of partially balanced designs, Ann. Math. Statist. vol. 30 (1959) pp. 21-38.

4. Alfred Brauer, On a new class of Hadamard determinants, Math. Z. vol. 58 (1953) pp. 219-225.

5. R. H. Bruck and H. J. Ryser, The nonexistence of certain finite projective planes, Canad. J. Math. vol. 1 (1949) pp. 88-93.

6. S. Chowla and H. J. Ryser, Combinatorial problems, Canad. J. Math. vol. 2 (1950) pp. 93-99.

7. E. C. Dade and K. Goldberg, The construction of Hadamard matrices, Michigan Math. J. vol. 6 (1959) pp. 247-250.

8. A. L. Dulmage and N. S. Mendelsohn, Coverings of bipartite graphs, Canad. J. Math. vol. 10 (1958) pp. 517-534.

9. — - The term and stochastic ranks of a matrix, Canad. J. Math. vol. 11 (1959) pp. 269-279.

10. C. J. Everett and G. Whaples, Representations of sequences of sets, Amer. J. Math. vol. 71 (1949) pp. 287-293.

11. L. R. Ford, Jr. and D. R. Fulkerson, $A$ simple algorithm for finding maximal network flows and an application to the Hitchcock problem, Canad. J. Math. vol. 9 (1957) pp. 210-218.

12. - Network flow and systems of representatives, Canad. J. Math. vol. 10 (1958) pp. 78-85.

13. D. R. Fulkerson, $A$ network flow feasibility theorem and combinatorial applications, Canad. J. Math. vol. 11 (1959) pp. 440-451.

14. ——, Zero-one matrices with zero trace, Pacific J. Math. vol. 10 (1960) pp. 831-836.

15. D. R. Fulkerson and H. J. Ryser, Widths and heights of $(0,1)$-matrices, Canad. J. Math., to appear. issue.

16. —- Traces, term ranks, widths and heights, IBM J., to appear in a special

17. David Gale, $A$ theorem on flows in networks, Pacific J. Math. vol. 7 (1957) pp. 1073-1082.

18. J. K. Goldhaber, Integral p-adic normal matrices satisfying the incidence equation, Canad. J. Math. vol. 12 (1960) pp. 126-133.

19. R. M. Haber, Term rank of 0, 1 matrices, Rend. Sem. Mat. Padova vol. 30 (1960) pp. 24-51.

20. Marshall Hall, Jr., Projective planes, Trans. Amer. Math. Soc. vol. 54 (1943) pp. 229-277.

21. - An existence theorem for Latin squares, Bull. Amer. Math. Soc. vol. 51 (1945) pp. 387-388.

22. - Cyclic projective planes, Duke Math. J. vol. 14 (1947) pp. 1079-1090.

23. - Distinct representatives of subsets, Bull. Amer. Math. Soc. vol. 54 (1948) pp. 922-926.

24. — - Projective planes and related topics, California Institute of Technology, 1954. 
25. - A survey of difference sets, Proc. Amer. Math. Soc. vol. 7 (1956) pp. 975-986.

26. - Some aspects of analysis and probability, New York, Wiley, 1958, pp. 35-104.

27. - The theory of groups, New York, Macmillan, 1959.

28. Marshall Hall and H. J. Ryser, Cyclic incidence matrices, Canad. J. Math. vol. 3 (1951) pp. 495-502.

29. - Normal completions of incidence matrices, Amer. J. Math. vol. 76 (1954) pp. 581-589. 26-30.

30. P. Hall, On representatives of subsets, J. London Math. Soc. vol. 10 (1935) pp.

31. Paul R. Halmos and Herbert E. Vaughan, The marriage problem, Amer. J. Math. vol. 72 (1950) pp. 214-215.

32. G. H. Hardy, J. E. Littlewood, and G. Pólya, Inequalities, Cambridge, University Press, 1952.

33. P. J. Higgins, Disjoint transversals of subsets, Canad. J. Math. vol. 11 (1959) pp. $280-285$.

34. Alan J. Hoffman, Some recent applications of the theory of linear inequalities to extremal combinatorial analysis, Proceedings of Symposia in Applied Mathematics, vol. 10, Amer. Math. Soc., 1960, pp. 113-128.

35. A. J. Hoffman and H. W. Kuhn, Systems of distinct representatives and linear programming, Amer. Math. Monthly vol. 63 (1956) pp. 455-460.

36. A. J. Hoffman, M. Newman, E. G. Straus, and O. Taussky, On the number of absolute points of a correlation, Pacific J. Math. vol. 6 (1956) pp. 83-96.

37. D. R. Hughes, Regular collineation groups, Proc. Amer. Math. Soc. vol. 8 (1957) pp. 165-168.

38. - Collineations and generalized incidence matrices, Trans. Amer. Math. Soc. vol. 86 (1957) pp. 284-296.

39. — Generalized incidence matrices over group algebras, Illinois J. Math. vol. 1 (1957) pp. 545-551.

40. Dénes König, Theorie der endlichen und unendlichen Graphen, New York, Chelsea, 1950.

41. H. B. Mann, Analysis and design of experiments, New York, Dover, 1949.

42. H. B. Mann and H. J. Ryser, Systems of distinct representatives, Amer. Math. Monthly vol. 60 (1953) pp. 397-401.

43. Marvin Marcus, On subdeterminants of doubly stochastic matrices, Illinois J. Math. vol. 1 (1957) pp. 583-590.

44. Marvin Marcus and Morris Newman, On the minimum of the permanent of a doubly stochastic matrix, Duke Math. J. vol. 26 (1959) pp. 61-72.

45. N. S. Mendelsohn and A. L. Dulmage, Some generalizations of the problem of distinct representatives, Canad. J. Math. vol. 10 (1958) pp. 230-241.

46. R. F. Muirhead, Some methods applicable to identities and inequalities of symmetric algebraic functions of $n$ letters, Proc. Edinburgh Math. Soc. vol. 21 (1903) pp. 144-157.

47. Paul J. Nikolai, Permanents of incidence matrices, Math. Comput. vol. 14 (1960) pp. 262-266.

48. Oystein Ore, Graphs and matching theorems, Duke Math. J. vol. 22 (1955) pp. 625-639.

49. R. E. A. C. Paley, On orthogonal matrices, J. Math. Phys. vol. 12 (1933) pp. 311-320. 
50. E. T. Parker, On collineations of symmetric designs, Proc. Amer. Math. Soc. vol. 8 (1957) pp. 350-351.

51. Günter Pickert, Projective Ebenen, Berlin, Springer-Verlag, 1955.

52. R. Rado, Factorization of even graphs, Quart. J. Math. Oxford vol. 20 (194.9) pp. 95-104.

53. H. J. Ryser, A note on a combinatorial problem, Proc. Amer. Math. Soc. vol. 1 (1950) pp. 422-424.

54. - A combinatorial theorem with an application to Latin rectangles, Proc. Amer. Math. Soc. vol. 2 (1951) pp. 550-552.

55. - Matrices with integer elements in combinatorial investigations, Amer. J. Math. vol. 74. (1952) pp. 769-773.

56. — Geometries and incidence matrices, Slaught Memorial Papers No. 4, Math. Assn. Amer., 1955, pp. 25-31.

57. — - Maximal determinants in combinatorial investigations, Canad. J. Math. vol. 8 (1956) pp. 245-249.

58. - Combinatorial properties of matrices of zeros and ones, Canad. J. Math. vol. 9 (1957) pp. 371-377.

59. - , The term rank of a matrix, Canad. J. Math. vol. 10 (1958) pp. 57-65.

60. - Inequalities of compound and induced matrices with applications to combinatorial analysis, Illinois J. Math. vol. 2 (1958) pp. 240-253.

61. - Compound and induced matrices in combinatorial analysis, Proceedings of Symposia in Applied Mathematics, vol. 10, Amer. Math. Soc., 1960, pp. 149-168.

62. — - Traces of matrices of zeros and ones, Canad. J. Math. vol. 12 (1960) pp. $463-476$.

63. - Combinatorial designs, to appear in a special publication of Math. Assn. Amer.

64. Robert Silverman, A metrization for power sets with applications to combinatorial analysis, Canad. J. Math. vol. 12 (1960) pp. 158-176.

65. James Singer, $A$ theorem in finite projective geometry and some applications to number theory, Trans. Amer. Math. Soc. vol. 43 (1938) pp. 377-385.

66. L. A. Skornyakov, Projective planes, Amer. Math. Soc. Translation No. 99, Providence, 1953.

67. P. V. Sukhatme, On bipartitional functions, Trans. Philos. Roy. Soc. London Ser. A vol. 237 (1938) pp. 375-409.

68. M. F. Tinsley, Permanents of cylic matrices, Pacific J. Math. vol. 10 (1960) pp. 1067-1082.

69. Olga Taussky Todd, Matrices of rational integers, Bull. Amer. Math. Soc. vol. 66 (1960) pp. 327-345.

70. Oswald Veblen and W. H. Bussey, Finite projective geometries, Trans. Amer. Math. Soc. vol. 7 (1906) pp. 241-259.

71. B. L. van der Waerden, Aufgabe 45, Jber. Deutsch. Math.-Verein. vol. 35 (1926) p. 117.

72. J. W. Williamson, Hadamard's determinant theorem and the sum of four squares, Duke Math. J. vol. 11 (1944) pp. 65-81.

73. - Note on Hadamard's determinant theorem, Bull. Amer. Math. Soc. vol. 53 (1947) pp. 608-613.

The Ohio State University 